\title{
Learning about the Scientific Method in an International Context: An Experiment with the Stroop Task
}

\author{
De Koning I, Jörg A, Homan J, Kancans Envall O, Landtmeters J, Pihl L, Smulders J, Stukje F, van der Voort D, van Goethem I, van Kastel \\ C and Moret-Tatay $C^{*}$
}

Catholic University of Valencia, Spain

\begin{abstract}
Science is not limited to researchers but it is mainly studied at the University. In this way, the internationalization of Higher Education has brought an enriching perspective to the topic that is addressed. Here, we came across a challenging scenario of learning the particular Stroop paradigm. A group of Erasmus students share its curiosity about their own differences in their performance, which fosters the present work. It is aimed at nurturing their (and other people's) learning during their early career. Therefore, an approximation to the scientific method was followed by them, which is described in the present work. Finally, a glossary of 18 terms was listed during the exercise. We hope this can be used as a motivating material for putting theory into practice.
\end{abstract}

Keywords: Scientific method; Researchers; International context

\section{Introduction}

Science is not limited to researchers. This might be one of the main mottos in the TEDTalk by the neuroscientist Beau Lotto. Here, the researcher shares their findings after working and carrying out a research project with school children. As a matter of fact, to assume that the practice of science is only available to an elite group goes against the pillars of science. However, the opportunities for junior and veteran researchers seem to be up for debate. Can everyone do real science then? Lotto tries to answer this question [1] by showing how even a small question can lead to a great discovery, but more precisely, that anyone can discover something new given the opportunity. Furthermore, this work was published in a peer-reviewed journal, Biology Letters. Fortunately, the scientific journals seem to be aware of how difficult it is for junior researchers to have the chance to communicate their results. Not surprisingly, some initiatives have been created to make them visible in the field, e.g., the increasing call for papers in some journals for this profile.

Focusing on the scientific method, this seems to be mainly studied at the University level. Bear in mind that this is a complex concept as it can vary greatly, even if it is characterized by a spiral of steps with regards to systematic observation and experimentation, inductive and deductive reasoning and the testing of hypotheses and underlying theories. In this way, the internationalization of Higher Education and its consequent student mobility provides an enriching perspective in this area. In addition, we consider that the inherent research in the topic might lead to a vast number of challenging questions on the analysis of international learning. Therefore, the aim of this work is to develop a broad and comprehensive learning of an experimental paradigm for the university students $[2,3]$. This work is the product of the work carried out by Erasmus students and the paradigm selected was the Stroop paradigm and its effects. Bear in mind that this is one of the most-studied phenomena in psychology. This particular paradigm requires the presentation of different stimuli in a particular language (through it is also possible to find adaptations with numbers avoiding the use of words). Therefore, we came across a challenging scenario full of individual differences for the mobility students. The student's curiosity about their own differences in their performance fosters the present work that aims to nurture their (and other people's) scientific curiosity during the early stages of their career. Hence, the different steps, taking the scientific method as a reference, are followed by the international students, which are described in the present work. Finally, 18 scientific terms were examined during the development of the research, as listed in the Appendix.

\section{Learning about the Stroop effect}

Firstly, we made a revision in the literature of the Stroop effect. As a brief introduction, this was first published by John Ridley Stroop, in 1935 , from where it gets its name. This effect is referred to the conflict between the controlled processing and automatic processing. In its original setup, it was developed for a naming task, where the participants have to recognize the colour in which a word is presented (e.g., recognizing the colour or ink presentation), to inhibit reading what is written, which is also the name of a colour. Here, the term paradigm and effect were revised. With regards to this topic, attentional human cognitive processes can be divided into two main groups, among others in this complex field. They can either be automatic, in which the process is effortless, unintentional and involuntary, or they can be controlled, in which the person is consciously aware about the process, in which it does take effort and can be constrained by the amount of attention resources [4]. With these two distinctive groups in mind, it is very interesting to look at the Stroop effect. Since the brain can instantly understand the meaning of a written word as a result of habitual reading, it can be seen as an automatic process. In contrast, recognizing colours is not an automatic process, but more a controlled process. When you combine two inputs that are contradictive, in this case a written colour in another colour, there is latency in response. The theory behind this is that although the automatic reading does not need controlled attention, it does use enough attention resources to

*Corresponding author: Moret-Tatay C, Catholic University of Valencia, San Vicente Mártir, Spain, Tel: +34 9636374 12; E-mail: carmenmoret@gmail.com

Received June 27, 2016; Accepted September 23, 2016; Published September 26, 2016

Citation: De Koning I, Jörg A, Homan J, Kancans Envall O, Landtmeters J, et al. (2016) Learning about the Scientific Method in an International Context An Experiment with the Stroop Task. Abnorm Behav Psychol 2: 128. doi: 10.4172/2472-0496.1000128

Copyright: (c) 2016 De Koning I, et al. This is an open-access article distributed under the terms of the Creative Commons Attribution License, which permits unrestricted use, distribution, and reproduction in any medium, provided the original author and source are credited. 
Citation: De Koning I, Jörg A, Homan J, Kancans Envall O, Landtmeters J, et al. (2016) Learning about the Scientific Method in an International Context: An Experiment with the Stroop Task. Abnorm Behav Psychol 2: 128. doi: 10.4172/2472-0496.1000128

reduce the amount of attention that is accessible for colour information processing.

Focusing on selective attention and participating in experiments like the Stroop test is interesting for psychology students because they will learn about automatic processes in the brain. This is what happens in a situation like the Stroop test. It is difficult to focus attention on naming the colours instead of the words. Reading is an automatic process for most people and it is difficult for the brain to switch off a stimulus like this. Knowing about automatic processes and how they can affect a daily life situation, people realize that they still have to use their brain to focus on attention. Those situations with different kinds of stimuli (like the words and the colours in the Stroop test) will distract the brain from what is important. This effect will be measured in terms of cognitive processing - to be more precise, by reaction times (RTs) and accuracy.

\section{Observing and forming questions}

In this part the students were encouraged to use search engines in order to broaden their knowledge about the topic.

One of the most relevant parts of the scientific method is formulating and testing scientific hypotheses. After differentiating between dependent and independent variables, and between hypotheses and predictions in a lecture early in the semester, students were encouraged to formulate their hypothesis. Scientists (even the younger ones) want to incorporate these components into the basis of their experimental tests. These are generated from a previous collection of as much evidence as possible. At the end of the investigation, they will determine whether they are accepted or not. Furthermore, hypotheses must be defined in a manner that makes them open to testing and falsification. In this learning process, the international student developed the following scientific research questions. In this way, two concepts were visited: The null $\left(\mathrm{H}_{0}\right)$ and the alternative hypothesis $\left(\mathrm{H}_{1}\right)$ The first one was defined as a starting point that is assumed to be true about a mean population (or better to say, a parameter). Moreover, as pointed out, we examine what we think is wrong about the null hypothesis in an alternative hypothesis. The inclusion of the dependent and independent variables was advised in the hypothesis. The two main ones were:

-Reaction times will be higher for the interference conditions (e.g., red) compared to the congruent ones (e.g., blue)

-Reaction times will be higher for the L1 block compared to the L2

-Reaction times will be higher for the L1 block compared to the L3

\section{Conducting an Experiment}

As mentioned before, one of the cognitive tasks that have been used frequently to examine the role of interference is the Stroop task. In this task, individuals have to name the colour in which a word is presented, while ignoring what is written (the name of a colour). This traditional set-up has been widely modified. In particular, we were interested about an experimental set-up. Therefore, a computer-based modified version, based on the original Stroop test version, and this is the version that was carried out. This procedure was similar to several pieces of research in the literature.

\section{Participants}

A sample of 18 University students, volunteered to take part in the experiment (14 women and 4 men with an average age of 20.83 years and $\mathrm{SD}=1.26$ ). All of them were Erasmus students for one semester (Swiss 6\%, Hungarian 6\%, Swedish 11\%, Dutch 39\% and Italian
39\%). Therefore, their L2 was English and their L3 was Spanish. All the participants had normal vision or corrected to normal and did not report cognitive or neurological disorders.

\section{Materials}

The presentation of the stimuli and recorded response times were controlled by computers through the Windows software DMDX (Forster and Forster, 2003). In each trial, a fixation point (+) was presented for $500 \mathrm{~ms}$ in the center of the screen. Then the target stimulus was presented until the participants responded, with a maximum of $2500 \mathrm{~ms}$. Word stimuli in experiment I were red, blue and xxxx. Word stimuli in experiment II was the same as in experiment I, but presented in the mother tongue of the participant. Finally, word stimuli in experiment III was the same as in experiment I, but presented in Spanish: rojo (which means red), azul (which means blue) and xxxx. The stimuli were presented in lowercase 14-pt Courier in order to control word length.

\section{Design and data analysis}

A 3 (Congruent, Incongruent and Control Stroop conditions) $\times 3$ (English, Mother tongue and Spanish language) factorial design was used. Participants were required to identify the colour red by pressing one key, and colour blue by pressing another. The stimuli was presented in either red or blue lowercase letters, and there were three presentation conditions: i) Congruent-red displayed in red or congruent-blue displayed in blue, ii) incongruent-red displayed in blue or incongruent-blue displayed in red, and iii) Neutral-xxxx in red or blue. In the first place, classical variance analysis was carried out, employing the software SPSS 21. Response times less than 250 or superior to $1500 \mathrm{~ms}$ and incorrect responses were excluded (less than $4 \%$ of the data set). This cut-off point was adopted for consistency with earlier studies in the field.

In addition to the original version, which consists of the three subtasks mentioned above, our test only consisted of one task with the three subtasks combined. The participants did the test three times in a different language. Firstly in English, then in their native language and finally in Spanish [5]. Stroop task performance was found to be dependent on the congruency between colour and word meaning. The responses are slower and the number of errors is higher when the word and the colour are incongruent. The repeated measures analysis and its underlying assumption were revisited.

\section{Results}

The aim of this exercise was not a deep knowledge into statistical methods; instead, a humble step in the scientific method was expected. In this way, the statistical section was developed under an expert supervision. Aspects such as statistical assumptions were examined among others. The average of latencies for correct responses and error rates are presented in Figure 1 and Table 1 (these were employed to examine the direction of the effects). An analysis of variance (ANOVAs) was performed on response times and accuracy. As expected, RTs were higher for the incongruent condition, but this difference only approached the significance level: $\mathrm{F}_{(2,34)}=2.98 ; \mathrm{MSE}=1254.82 ; \eta^{2}$ $=0.149 ; \mathrm{p}=0.06$. Moreover, RTs were higher for the L1 language, however, this difference did not reach the significance level: $\mathrm{F}_{(2,34)}=2.17$; $\mathrm{MSE}=8372.19 ; \eta^{2}=0.113 ; \mathrm{p}=0.12$. No statistically significant differences were found for accuracy (all $\mathrm{F}<1$ ).

It is important to notice that the direction of the effects followed the underlying hypothesis, however, those were not statistically significant. 
Citation: De Koning I, Jörg A, Homan J, Kancans Envall O, Landtmeters J, et al. (2016) Learning about the Scientific Method in an International Context: An Experiment with the Stroop Task. Abnorm Behav Psychol 2: 128. doi: 10.4172/2472-0496.1000128
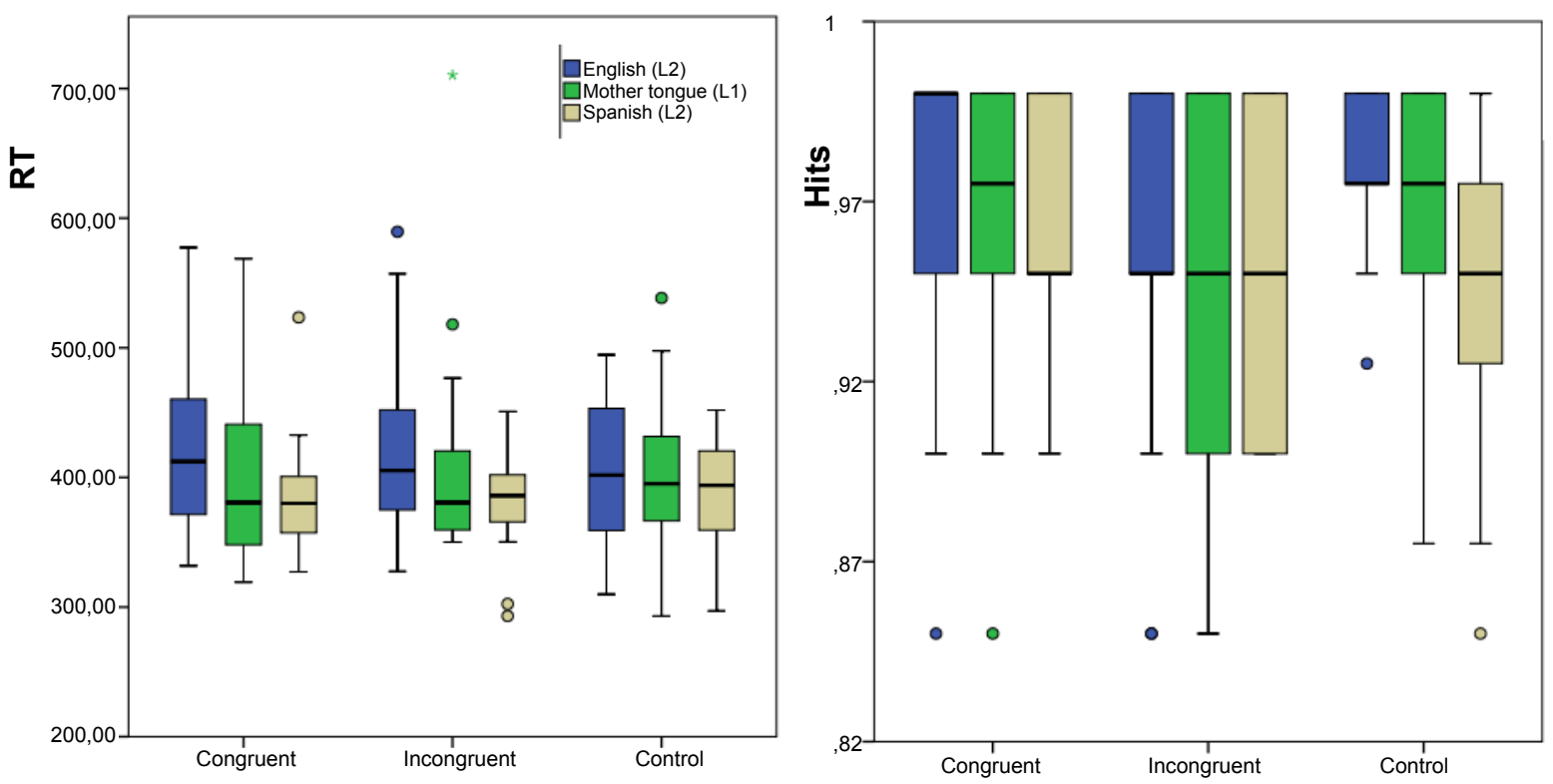

Figure 1: Box and whisker graph for the Stroop task with regards to language. Left side: The reaction times. Right side: The accuracy or hits.

\begin{tabular}{|c|c|c|c|c|}
\hline $\begin{array}{c}\text { Language } \\
\text { Condition }\end{array}$ & Congruent & Incongruent & Control \\
\hline L1 & $\begin{array}{c}\text { RT } \\
(\text { Hits \%) }\end{array}$ & $\begin{array}{c}420.43 \\
(0.97)\end{array}$ & $\begin{array}{c}433.12 \\
(0.95)\end{array}$ & $\begin{array}{c}410.25 \\
(0.97)\end{array}$ \\
\hline & SD & 66.39 & 117.71 & 54.90 \\
\hline L2 & RT & 403.21 & 415.92 & 401.26 \\
& (Hits \%) & $(0.96)$ & $(0.95)$ & $(0.96)$ \\
\hline & SD & 72.47 & 87.98 & 56.20 \\
\hline L3 & RT & 386.92 & 389.87 & 377.63 \\
& (Hits \%) & $(0.95)$ & $(0.95)$ & $(0.94)$ \\
\hline & SD & 44.62 & 41.68 & 39.14 \\
\hline
\end{tabular}

a higher sample size. Furthermore, the fatigue effect (when participant becomes tired or bored while performing a sequence of tasks) was also visited. Finally, the difference between languages (e.g., the grade of the transparency of a language), the particular expertise in their L2 and L3, among other variables, were also suggested as a source of error. Even if this was highly reasonable to be taken into account, it was not developed in the present study. These are crucial questions, but it was left apart in order to focus on the basic questions mentioned before.

What we have presented here is an example of putting theory into practice in an international context. We believe that study material should come from students' interest and questions, more than teachers or researchers. Moreover, learning science on a theoretical level exclusively might promote students' loss of interest. In particular, Frostada, Pijlb and Mjaavatn found that teacher support and loneliness were predictors of dropping out of or carrying on with studies. This exercise aims to offer an example where students can develop their role as a researcher by examining most of the steps that occur within the scientific method. Furthermore, it seems fundamental to emphasize the meaning of science and its role in our lives on a practical level. But this action seems not only to be of interest of the students, it also for the teachers. For example, a study developed by Brodie and Hudson showed the benefitial effect $s$ of approaching science. In this case, some pre-doctoral and post-doctoral students volunteered to spend several days in the schools. After evaluating its effects, they continued to popularize science in this field. We hope these type of activities help them to address the real scope and limitations of science and scientists first hand. As we mentioned, science is for everyone, our students included.

\section{Acknowledgement}

We would like to thank Thomas Irvin for his invaluable help and revision of the manuscript.

\section{References}

1. Baron RS (1986) Distraction-conflict theory: Progress and problems. Academic Press, New York. 
Citation: De Koning I, Jörg A, Homan J, Kancans Envall O, Landtmeters J, et al. (2016) Learning about the Scientific Method in an International Context: An Experiment with the Stroop Task. Abnorm Behav Psychol 2: 128. doi: 10.4172/2472-0496.1000128

Page 4 of 4

2. Besner D (2001) The myth of ballistic processing: Evidence from Stroop's paradigm. Psychon Bull Rev 8: 324-330.

3. Besner D, Stolz JA, Boutilier C (1997) The Stroop effect and the myth of automaticity. Psychon Bull Rev 4: 221-225.

4. Broadbent DE (1971) Decision and stress. Academic Press, New York.
5. Cacioppo JT (2002) Social neuroscience in perspective: Understanding the pieces contributes to understanding the whole and vice versa. Am Psychol 57: 819-831.

6. Cacioppo JT, Berntson GG, Lorig TS, Norris CJ, Rickett E, et al. (2003) Just because you're imaging the brain doesn't mean you can stop using your head: A primer and set of first principles. J Pers Soc Psychol 85: 650-661. 